\title{
Manipulation CheCKING THE MUNCHiEs: VALIDATING SELF-REPORTED DiETARY BEHAVIORS DURING CANNABIS INTOXICATION
}

\author{
Jessica S. Kruger ${ }^{1}$, Alexis Blavos ${ }^{2}$, Thomas S. Castor ${ }^{3}$, Amy J. Wotring ${ }^{3}$, Victoria R. \\ Wagner-Greene ${ }^{3}$, Tavis Glassman ${ }^{3}$, Daniel J. Kruger ${ }^{4}$ \\ 1Department of Community Health and Health Behavior, SUNY at Buffalo, New York, USA \\ 2Department of Health, SUNY Cortland, New York, USA \\ 3 University of Toledo, $\mathrm{OH}$, USA \\ 4Population Studies Center, University of Michigan, Ann Arbor, MI, USA
}

jskruger@buffalo.edu

\begin{abstract}
The prevalence and rate of cannabis use will likely increase as the relevant legal framework becomes more permissive across many municipalities. All policies and practices should be informed by scientific evidence and the public health framework for cannabis users may benefit from research and interventions promoting harm reduction. Naturalistic observations are particularly valuable for understanding patterns of human behavior and can complement and validate information collected through other methods, such as self-report surveys. Observational research may be especially valuable for addressing potentially controversial topics where behaviors are socially stigmatized and/or illegal. The current study examined the relationship between self-reported dietary behaviors and food incentive choices among individuals intoxicated with cannabis. Participants at a cannabis decriminalization advocacy event completed brief surveys on cannabis use and dietary habits. Survey administrators offered an incentive choice of a food items; fruit and chips/crisps. Researchers documented choices of food incentives and three trained coders categorized self-reported dietary habits. Participants $(N=275)$ reported eating unhealthy food (77\%), eating healthy food (23\%), and avoiding consuming food when intoxicated (7\%). Participants also reported eating more food when intoxicated with cannabis and being more likely to eat unhealthy food when intoxicated compared to at other times. Food incentive choices predicted self-reported habits for both consuming healthy and unhealthy foods. Observational results validated self-reported dietary habits and confirmed common stereotypes.
\end{abstract}

Keywords: Observational research, Munchies, Cannabis, Diet, Validation. 


\section{INTRODUCTION}

Naturalistic observation involves the direct observation of behaviors in their natural setting, with no intervention, deception, or manipulation by a researcher or research confederate (Tinbergen, 1963). The real-life context differentiates naturalistic observation from experimental research, where artificial environments are created to control for spurious and potentially confounding factors. Naturalistic observations are valuable for investigating potentially controversial topics where related behaviors are socially stigmatized and/or illegal and may be superior to self-report survey results in their utility to inform policy-making. In addition to responses influenced by social desirability, survey self-reports may be subject to recall biases, as well as contextdependent memory. Observational research has previously provided insights on healthrelated activities, including on dietary behaviors (Kruger, Castor, \& Kruger, 2016). The current study examined the relationship between self-reported dietary behaviors and food incentive choices among individuals intoxicated with cannabis.

Cannabis (colloquially "marijuana") is a flowering plant containing psychoactive compounds that has been used by humans for over 5,000 years (Lucas, 2012). Cannabisbased remedies were popular for treatment of a wide variety of ailments in western nations during the early 19th Century, though they were criminalized in the early 20th Century (Grinspoon \& Bakalar, 1997). Beginning in the late 20th Century, cannabis related laws have been reformed to allow for medicinal and recreational use in many municipalities such as Australia, Canada, Colombia, Czech Republic, Bolivia, Mexico, Netherlands, Portugal, and South Africa. As of January 2018, over 21\% of Americans lived where recreational cannabis is legal at the state level, and less than $2 \%$ of the U.S. population live in states with full cannabis prohibition (NCSL, 2017). Cannabis use in the United States has risen rapidly over the past decade and prevalence rates have recently surpassed those for tobacco use (Azofeifa, Mattson, \& Grant, 2016).

As the legal framework surrounding cannabis becomes more permissive and use prevalence rates increase, the population of cannabis users will grow. Cannabis users have unique health issues, which have not been adequately studied (National Academies of Sciences, Engineering, and Medicine, 2017). Current public health efforts in areas such as the United States typically focus on cannabis prohibition and abstinence. A host of cannabis-related issues will have a greater impact on users and society as the prevalence of cannabis use increases. An evidence-based approach would be beneficial for shaping cannabis-related policies and practices to reduce harm and maximize benefits to individuals and society (Erickson, Riley, Cheung, \& O'Hare, 2015).

Researchers and practitioners must integrate a better understanding of the health behaviors of cannabis users and the related health issues into their work to better serve this unique and growing population. Treatment and prevention strategies, including riskreduction techniques, need to be tailored to meet the unique properties of this population. Previous studies have explored the mechanisms of what many call the "munchies"- compulsions to eat, stimulated by the active ingredients of cannabis, the cannabinoids. Cannabinoids can act at several brain regions to stimulate food intake (Cota et al., 2003; Kirkham, 2009; Soria-Gómez et al., 2014). Researchers have documented a significant increase in consumption of foods that are high in carbohydrates when under the influence of cannabis (Foltin et al., 1988; Mohs, Watson, 
\& Leonard-Green, 1990; Smit \& Crespo, 2001). Studies dating as far back as the 1970s have found that cannabis use increases appetite and food intake (Kirkham, 2005), however, behavioral studies were conducted in residential laboratories (e.g., Foltin, Fischman, \& Byrne, 1988; Greenberg, Kuehnle, Mendelson, \& Bernstein, 1976) rather than naturalistic settings.

\section{Current Study}

The current study explored the relationship between self-reported food consumption and dietary choices during cannabis intoxication. Hypotheses: (H1) participants will report eating more food when intoxicated with cannabis compared to at other times; (H2) the majority of participants will report eating unhealthy food when intoxicated with cannabis; (H3) participants will reported being more likely to eat more unhealthy foods when intoxicated with cannabis compared to at other times; (H4) the majority of participants will choose chips/crisps as a food incentive over a fruit incentive when intoxicated with cannabis; and (H5) food incentive choices will predict self-reported habits for healthy (H5a) and unhealthy (H5b) food consumption when intoxicated with cannabis.

\section{METHODS}

\section{Context}

This project was reviewed by the Health Sciences and Behavioral Sciences Institutional Review Board at the University of Michigan prior to data collection. Individuals attending the 2016 Hash Bash, an annual public forum on a university campus in a Midwestern U.S. state advocating for the reform of cannabis laws, were invited to participate. There were 275 surveys completed, 26 individuals withdrew from the study and their incomplete surveys were not included in analyses. One respondent who had never used cannabis was excluded from analyses. Participants were 65\% men, 35\% women, were 25 years of age on average $(S D=10$, range $=18-88$ years), had 13 years of education on average ( $\mathrm{SD}=2$, range $=3-20$ years), and $41 \%$ were current college students. Self-reported races/ethnicities (inclusive) were White (85\%), Black/African American (11\%), American Indian or Alaska Native (10\%), Hispanic/Latino (7\%), and Asian (3\%). In 2010, American Indian or Alaska Natives comprised $0.6 \%$ of the state population (U.S. Census Bureau, 2012). The relatively high proportion of individuals identifying as American Indian or Alaska Native may be due to a Native American cultural event held locally during the same weekend.

The research team was trained on the survey administration process, including informed consent and debriefing, prior to data collection. The research team set up a table at the edge of academic quadrangle adjacent to the primary and shortest walking path to the downtown business district. Researchers verbally invited potential participants in the immediate area to complete the survey, confirming that they met the eligibility criteria of being 18 years of age or older and identifying as a current cannabis user. After informed consent was established, participants were provided a hard copy 
survey, clipboard, and pen. Participants were offered the choice of a small food item when the survey was completed, either a fruit or a bag of chips/crisps.

The survey included specific questions about cannabis use, food consumption, and demographics, using colloquial terminology. Questions included: "What do you typically eat when you are high on marijuana?" with an open-ended response box. This was followed by the items "Are you more or less likely to eat these foods when you are high on marijuana compared to other times?" and "How much more or less do you eat when you are high on marijuana compared to other times?," answered with a five-point response scale (Much less, Somewhat less, About the same, Somewhat more, Much more). Demographic items included age, sex, race/ethnicity, educational attainment, and student status. We examined the potential influence of the degree of cannabis intoxication on the other study variables. Participants were asked, "How high do you feel right now?" and could select from Not at all, Somewhat, Moderately, Very, or Extremely. Researchers documented healthy food incentive choices (fruit or no food item requested) and unhealthy choices (refined carbohydrates; chips/crisps).

Three study team members with graduate level training in health and nutrition independently coded open-ended responses. Participants could list multiple items, each item was coded in a separate variable, so an individual participant could be documented as consuming healthy foods, unhealthy foods, both healthy and unhealthy foods, or neither. Coding discrepancies (less than 5\%) were resolved by discussion among the coders. Quantitative data analysis included descriptive statistics and inferential statistics to examine study hypotheses.

\section{RESULTS}

Participants reported eating more food when intoxicated with cannabis compared to at other times, $t_{(271)}=10.92, p<.001, d=3.55$, supporting Hypothesis 1 . Most $(77 \%)$ participants reported eating unhealthy foods when intoxicated with cannabis, supporting Hypothesis 2. Also, 23\% reported eating healthy food when intoxicated, while $7 \%$ reported avoiding food when intoxicated. Participants reported being more likely to eat unhealthy food when intoxicated with cannabis compared to at other times, $r(217)=.636$, $p<.001$, supporting Hypothesis 3 . There was no difference in the likelihood of eating healthy foods when intoxicated with cannabis compared to at other times, $r_{(215)}=.081, p$ $=.238$. The majority (61\%) of participants chose the chips/crisps (95\% Confidence Interval: $55 \%-67 \%)$ over the fruit (32\%) or no food item (7\%), supporting Hypothesis 4. Those who reported eating healthy foods when intoxicated with cannabis were more likely to choose fruit as an incentive, $r_{(213)}=.227, p<.001$, and less likely to choose chips as an incentive, $r_{(213)}=.260, p<.001$, supporting Hypothesis 5a. Those who reported eating unhealthy foods when intoxicated with cannabis were less likely to choose fruit as an incentive, $r_{(213)}=-.176, p=.010$, and more likely to choose chips as an incentive, $r_{(213)}$ $=.206, p=.003$, supporting Hypothesis $5 \mathrm{~b}$. 
The HC-Holm procedure (see Toothaker, 1993) was used to verify that the pattern of results was consistent when assessed with an Error-Rate Family-Wise (ERFW) of .05. At the time of the survey, participants reported feeling extremely (14\%), very (22\%), moderately (29\%), somewhat (16\%), and not at all high (19\%). There were no relationships between current cannabis intoxication and study variables.

\section{DISCUSSION}

Current public health efforts typically focus on abstinence promotion and investigations of possible deleterious effects of cannabis use. Efforts to prevent cannabis use may still be appropriate for certain populations, such as minors or pregnant women. However, the current public health framework focusing predominantly on cannabis abstinence is too narrow in scope. Given the growing use of cannabis for treatment of health issues, as well as substantial recreational use, a shift towards minimizing harm and risk and maximizing benefits would be beneficial. Risk reduction strategies represent a promising alternative approach to minimizing harm for people who choose to use cannabis for medicinal and/ or recreational purposes (Erickson et al., 2015). Science should inform all policy, especially in areas where personal values may differ. Those working in the fields of public health and medicine have an obligation to reduce harm and maximize benefits to the health of individuals and society.

Although considerable research has investigated health risks associated with cannabis use, this is the first systematic naturalistic study investigating food choices during cannabis intoxication. Participants' choices were consistent with self-reported dietary behavior during previous cannabis intoxication. Those who reported making healthy food choices were more likely to make a healthy food choice, those who reported making unhealthy food choices were more likely to make an unhealthy food choice. Participants reported eating more food when under the influence of cannabis compared to at other times, consistent with previous research on dietary self-reports (Pertwee, 2014). Additionally, the majority of participants in this study self-reported that they are likely to eat more food and choose less healthy food when intoxicated, consistent with the majority of food choices observed. When individuals use cannabis, their judgements and decisions are affected by cannabis intoxication (Fisk, Morley, Hadjiefthyvoulou, \& Montgomery, 2014). This study helps confirm the relationships between cannabis intoxication and dietary decisions.

\section{Limitations}

Given the nature of self-reported data, responses may have included recall bias-due to drug use-and some participants may have experienced difficulty remembering how often they used marijuana and the related consequences. However, previous research has demonstrated links between impaired memory and cannabis intoxication but these effects appear to be for primarily for working memory rather than long-term memory (Miller, McFarland, Cornett, \& Brightwell, 1977; Morgan, Schafer, Freeman, \& Curran, 2010). Also, state-dependent memory may actually enhance recall for behaviors associated with cannabis intoxication (Overton, 1984). Some participants may have 
responded with socially desirable answers to appease perceived researcher expectations or may have been motivated to make food incentive choices consistent with their selfreports. This study included a convenience sample of regional cannabis users during a cannabis decriminalization advocacy event. Thus, the findings may not generalize to other locations or populations. With all cross-sectional research there are limitations, including completely understanding the causal relationships between cannabis intoxication and food choices. It is possible that some participants tend to make unhealthy choices, whether or not they are under the influence of cannabis. Further, some participants may prefer healthy foods, just not the ones provided at this study, same with the unhealthy choices.

\section{Conclusion}

Overall, this study demonstrates the value of observational studies for understanding health-related behaviors. All public policies and health-promoting initiatives should be informed by scientific evidence. The convergent evidence from multiple methodologies validated self-reported dietary habits. As more municipalities decriminalize or legalize cannabis, the need for tailored nutrition education among the population of cannabis users grows. Additional intervention research is needed to understand effective means of modifying unhealthy dietary choices made while intoxicated with cannabis.

\section{REFERENCES}

Azofeifa, A., Mattson, M. E., \& Grant, A. (2016). Monitoring marijuana use in the United States: challenges in an evolving environment. Journal of the American Medical Association, 316, 1765-1766. DOI

Cota, D., Marsicano, G., Lutz, B., Vicennati, V., Stalla, G., Pasquali, R., \& Pagotto, U. (2003). Endogenous cannabinoid system as a modulator of food intake. International Journal of Obesity, 27, 289. DOI

Erickson, P.G., Riley, D.M., Cheung, Y.W., \& O'Hare, P.A. (2015). Harm reduction: A new direction for drug policies and programs. Toronto, ON: University of Toronto Press. $\underline{\mathrm{DOI}}$

Fisk, J.E., Morley, A.M., Hadjiefthyvoulou, F., \& Montgomery, C. (2014). Reasoning deficits among illicit drug users are associated with aspects of cannabis use. Cognitive Processing, 15(4), 523-534. DOI

Foltin, R. W., Fischman, M. W., \& Byrne, M. F. (1988). Effects of smoked marijuana on food intake and body weight of humans living in a residential laboratory. Appetite, 11(1), 1-14. DOI

Greenberg, I., Kuehnle, J., Mendelson, J. H., \& Bernstein, J. G. (1976). Effects of marihuana use on body weight and caloric intake in humans. Psychopharmacology, 49(1), 79-84. DOI

Grinspoon, L. \& Bakalar, J.B. (1997). Marihuana: The forbidden medicine. New Haven, CT: Yale University Press.

Kirkham, T.C. (2005). Endocannabinoids in the regulation of appetite and body weight. Behavioural Pharmacology, 16(5-6), 297-313. DOI

Kirkham, T.C. (2009). Cannabinoids and appetite: food craving and food pleasure. International Review of Psychiatry, 21(2), 163-171. DOI 
Kruger, J.S., Castor, T., \& Kruger, D.J. (2016). Caffeine and calorie consumption at a college campus coffee café. Human Ethology Bulletin, 31(1), 74-83. DOI

Lucas, P. (2012). Cannabis as an adjunct to or substitute for opiates in the treatment of chronic pain. Journal of Psychoactive Drugs, 44(2), 125-133. DOI

Miller, L.L., McFarland, D., Cornett, T.L., \& Brightwell, D. (1977). Marijuana and memory impairment: effect on free recall and recognition memory. Pharmacology Biochemistry and Behavior, 7(2), 99-103. DOI

Mohs, M. E., Watson, R. R., \& Leonard-Green, T. (1990). Nutritional effects of marijuana, heroin, cocaine, and nicotine. Journal of the American Dietetic Association, 90(9), 1261-1267.

Morgan, C.J., Schafer, G., Freeman, T.P., \& Curran, H.V. (2010). Impact of cannabidiol on the acute memory and psychotomimetic effects of smoked cannabis: naturalistic study. The British Journal of Psychiatry, 197(4), 285-290. DOI

National Conference of State Legislatures (NCSL). (2017). Marijuana overview. Washington, DC: Author. Retrieved from http://www.ncsl.org/research/civil-and-criminal-justice/ marijuana-overview.aspx

Overton, D.A. (1984). State dependent learning and drug discriminations. In L.L. Iversen, S.D. Iversen, \& S. Snyder (Eds.), Handbook of Psychopharmacology Volume 18: Drugs, Neurotransmitters, and Behavior (pp. 59-127). New York: Springer. DOI

Pertwee, R.G. (2014). Handbook of cannabis. Oxford, UK: Oxford University Press. DOI

National Academies of Sciences, Engineering, and Medicine. (2017). The health effects of cannabis and cannabinoids: the current state of evidence and recommendations for research. Washington, DC: National Academies Press. DOI

Smit, E., \& Crespo, C. J. (2001). Dietary intake and nutritional status of US adult marijuana users: results from the Third National Health and Nutrition Examination Survey. Public health nutrition, 4(3), 781-786. DOI

Soria-Gómez, E., Bellocchio, L., Reguero, L., Lepousez, G., Martin, C., Bendahmane, M., Ruehle, S., Remmers, F., Desprez, T., Matias, I., Wiesner, T., Cannich, A., Nissant, A., Wadleigh, A., Pape, H.C., Chiarlone, A.P., Quarta, C., Verrier, D., Vincent, P., Massa, F., Lutz, B., Guzmán, M., Gurden, H., Ferreira, G., Lledo, P.M., Grandes, P., \& Marsicano, G. (2014). The endocannabinoid system controls food intake via olfactory processes. Nature Neuroscience, 17, 407-415. DOI

Tinbergen, N. (1963). On aims and methods of Ethology. Zeitschrift für Tierpsychologie, 20, 410-433. DOI

Toothaker, L. E. (1993). Multiple comparison procedures. Newbury Park, CA: Sage. DOI

U.S. Census Bureau. (2012). 2010 Decennial Census. Washington, DC: U.S. Department of Commerce. 